\title{
A path-integral Monte Carlo study of a small cluster: The Ar trimer
}

\author{
R. Pérez de Tudela, M. Márquez-Mijares, ${ }^{\text {a) }}$ T. González-Lezana, ${ }^{\text {b) }}$ O. Roncero, \\ S. Miret-Artés, G. Delgado-Barrio, and P. Villarreal \\ Instituto de Física Fundamental, CSIC, Serrano 123, 28006 Madrid, Spain
}

(Received 11 November 2009; accepted 14 May 2010; published online 23 June 2010)

$\mathrm{The} \mathrm{Ar}_{3}$ system has been studied between $T=0 \mathrm{~K}$ and $T=40 \mathrm{~K}$ by means of a path-integral Monte Carlo (PIMC) method. The behavior of the average energy in terms of the temperature has been explained by comparison with results obtained with the thermal averaged rovibrational spectra estimated via: (i) a quantum mechanical method based on distributed Gaussian functions for the interparticle distances and (ii) an analytical model which precisely accounts for the participation of the dissociative continua $\mathrm{Ar}_{2}+\mathrm{Ar}$ and $\mathrm{Ar}+\mathrm{Ar}+\mathrm{Ar}$. Beyond $T \sim 20 \mathrm{~K}$, the system explores floppier configurations than the rigid equilateral geometry, as linear and $\mathrm{Ar}-\mathrm{Ar}_{2}$-like arrangements, and fragmentates around $T \sim 40 \mathrm{~K}$. A careful investigation of the specific heat in terms of a confining radius in the PIMC calculation seems to discard a proper phase transition as in larger clusters, in apparent contradiction with previous reports of precise values for a liquid-gas transition. The onset of this noticeable change in the dynamics of the trimer occurs, however, at a remarkably low value of the temperature in comparison with $\mathrm{Ar}_{n}$ systems formed with more $\mathrm{Ar}$ atoms. Quantum mechanical effects are found of relevance at $T \leq 15 \mathrm{~K}$, with both energies and radial distributions obtained with a quantum PIMC deviating from the corresponding classical results, thus precluding exclusively classical approaches for a precise description of the system at this low temperature range. (C) 2010 American Institute of Physics. [doi:10.1063/1.3445773]

\section{INTRODUCTION}

Finite clusters have been largely studied as ideal prototypes between molecules and bulk matter. The possible occurrence in microscopic molecular aggregates of properties originally observed in the bulk has motivated profound investigations on such systems. Thus, for example, apparent evidences of phase transitions in rare gas clusters, ${ }^{1}(\mathrm{Rg})_{n}$, and small systems ${ }^{2}$ have been analyzed in detail in the past forty years. Changes in quantities such as radial density functions, diffusion coefficients, total internal energy, and bond length distances as the temperature $T$ increases were originally interpreted as indications of a transition from a solidlike state to a liquidlike form for $(\mathrm{Rg})_{n}$ systems. ${ }^{3-8} \mathrm{Ex}-$ perimental works, on the other hand, have not provided conclusive evidences in support of the possibility of phase coexistence in such microclusters. ${ }^{9-18}$

The onset of this nonrigid dynamics in Rg clusters has also been studied by identifying the solidlike cluster with a conventional molecule displaying a near-rigid behavior and the liquidlike form with a very nonrigid molecule which can explore the different energetically available potential energy minima. The onset of melting in the cluster is therefore related to the occurrence of frequent passages through the potential minima wells associated to the possible stable isomers. $^{1,19-21}$

The notion of phase transitions in very small clusters,

\footnotetext{
a) Also at Instituto Superior de Tecnologías y Ciencias Aplicadas, Ave. Salvador Allende y Luaces Quinta de Los Molinos, Plaza, La Habana 10600, Cuba.

${ }^{b)}$ Electronic mail: tglezana@imaff.cfmac.csic.es.
}

however, contradicts the seemingly well accepted dogma which requires systems to possess a large number of particles to show a true sharp phase change. ${ }^{22}$ This issue has been discussed, for three-atom systems, with analogs between phases and energy localization modes in a molecule. ${ }^{23}$ In this sense, trimers, exhibiting only an energy potential minimum [for the equilateral geometry in the case, for instance, of $\mathrm{Ar}_{3}$ or for a linear arrangement in the case of ionic trimers such as $\mathrm{Kr}_{3}^{+}$(Ref. 24) and $\mathrm{Ar}_{3}^{+}$(Ref. 25)] would constitute an extreme case of such possible behavior. For $\mathrm{Ar}_{3}$, dynamics in the equilateral triangle and collinear regions have been studied via the corresponding energy levels and eigenfunctions for a zero total angular momentum. ${ }^{26}$ Although the mode softening and diffusion characteristics as its energy increases could be interpreted as dynamical properties of a system passing from a rigid to a nonrigid form, this assumption may certainly constitute an oversimplification of the melting transition in larger systems. ${ }^{5,21,27,28}$ Etters and Kaelberer, ${ }^{6}$ however, reported a liquid-gas transition temperature of $20 \mathrm{~K}$ for the Ar trimer. Interestingly, Leitner et al. ${ }^{29}$ reported a not very different effective temperature, $T=28 \mathrm{~K}$, for the beginning of diffusive motion (compared by the authors with a phase change) within the $\mathrm{Ar}_{3}$ cluster as the atoms reach the saddle of the linear configuration.

Despite some other theoretical methods have been employed to study $(\mathrm{Rg})_{n}$ clusters, such as molecular dynamics (MD) simulations, most of our understanding of the thermodynamics of these complexes nowadays comes from Monte Carlo (MC) calculations. In particular, finite temperature path-integral MC (PIMC) methods have been applied for clusters such as $\operatorname{Rg}_{6},{ }^{30-32} \operatorname{Rg}_{7},{ }^{33-35} \operatorname{Rg}_{13},{ }^{30-32,36-40} \operatorname{Rg}_{19},{ }^{37}$ 
and $\operatorname{Rg}_{38} \cdot{ }^{41}$ For three-body (3B) clusters, the list of previous works is much more reduced. The work by Rick et al., ${ }^{35}$ which includes the energy of the Ne trimer as a function of $T$ up to $3 \mathrm{~K}$, constitutes, in this sense, the sole exception of a quantum mechanical (QM) PIMC study. Classical and parallel tempering $\mathrm{MC}$ methods were employed for the investigation on the thermodynamical properties of $\mathrm{Kr}_{3}^{+}$(Ref. 24) and $\mathrm{Ar}_{3}^{+},{ }^{25}$ respectively. Recently, Mandelshtam and co-workers have developed, as a complementary tool for PIMC studies of $\operatorname{Rg}_{n}$ clusters with $n \geq 39$ atoms, an approximative approach which employs variational Gaussian wave packets and MC sampling techniques. ${ }^{41-44}$ In the present study, we use a PIMC approach to investigate some of the thermodynamical properties of the Ar trimer.

Two other approaches, based on the thermal average of the rovibrational spectra for different values of the total angular momentum $J$, have been also employed. On one hand, bound state energies and wave functions for the rovibrational case $J \geq 0$ are calculated by means of a QM method, which employs distributed Gaussian functions (DGFs) in terms of the interparticle distances $R_{1}, R_{2}$, and $R_{3} \cdot{ }^{45,46}$ A preliminary comparison between the internal energy of the title system at low $T(T \leq 1 \mathrm{~K})$ calculated with a PIMC approach and the predictions of the DGF method has been reported recently. ${ }^{47}$ In this work we have extended the temperature range of the analysis. On the other hand, we employ a model based on two Morse potentials, centered at different equilibrium distances. The analytical expressions of both discrete and continuum spectra are employed to construct the partition functions of the corresponding Boltzmann average. Similar attempts for alternatives to the exact density of states, which was calculated with quasiclassical approaches, have been done in the past. ${ }^{27,48}$

A largely investigated issue regards the possible presence of QM effects on the thermal properties of Rg clusters. The comparison of classical and QM MC calculations of quantities such as energies, ${ }^{30,32,40,49}$ specific heats, ${ }^{32,40-42,50}$ root mean square fluctuations, ${ }^{28,32,33}$ scattering functions, ${ }^{31}$ correlation functions, ${ }^{30,32,33,41}$ and distributions of quench results on stable isomers ${ }^{35}$ reveals that, in general, at low temperature, the dynamics of these clusters is notably nonclassical. Similar studies have been undertaken in this work. In particular, we have addressed the comparative analysis of classical and QM energies and correlation functions by means of the PIMC approach.

The paper is organized as follows. In Sec. II we describe the theoretical methods employed here; results are shown in Sec. III and discussed in Sec. IV. Finally, the main conclusions of the present study will be presented in Sec. V.

\section{THEORY}

In this section we will present the PIMC method and the two approaches based on the calculation of the rovibrational spectra of the system: the DGF method and a model which employs analytical energy levels for two combined $\mathrm{Ar}-\mathrm{Ar}_{2}$ interactions.

The potential energy surface employed in the PIMC and DGF calculations is constructed as a sum of pairwise Morse potentials whose parameters, also used before to study the $\mathrm{Ar}_{3}$ system, ${ }^{51}$ are given by $D=99 \mathrm{~cm}^{-1}, \alpha=1.717 \AA^{-1}$, and $r_{0}=3.757 \AA$. No 3B terms have been considered.

\section{A. Path-integral Monte Carlo method}

The PIMC approach employed here has been described elsewhere. ${ }^{52,53}$ Briefly, the $3 \mathrm{~B}$ density matrix at a temperature $T$ is replaced by the product of $M$ density matrices at higher temperatures $M T$,

$$
\rho\left(\mathcal{R}_{1}, \mathcal{R}_{M+1} ; \beta\right)=\int d \mathcal{R}_{2} \cdots d \mathcal{R}_{M} \prod_{\alpha=1}^{M} \rho\left(\mathcal{R}_{\alpha}, \mathcal{R}_{\alpha+1} ; \tau\right)
$$

where $\beta=1 / k_{B} T$ and $\tau=\beta / M . \mathcal{R}_{\alpha}$ is the vector which collects the $3 N$ positions of the $N$ particles: $\mathcal{R}_{\alpha} \equiv\left\{\mathbf{r}_{1}^{\alpha}, \ldots, \mathbf{r}_{N}^{\alpha}\right\}$, $\mathbf{r}_{i}^{\alpha}$ being the position vector of the $i$ th $\mathrm{Ar}$ atom at the time slice or imaginary time $\alpha$. It is important ${ }^{52}$ to distinguish between $\tau$ and the iteration step, intrinsic to the movements of the paths in the PIMC calculation, hereafter noted as $t$. The internal energy can be obtained as the thermal average of the Hamiltonian $\hat{H}$ as

$\langle E(T)\rangle=Z^{-1} \int d \mathcal{R}_{1} \int d \mathcal{R}_{M+1}\left\langle\mathcal{R}_{M+1}|\hat{H}| \mathcal{R}_{1}\right\rangle \rho\left(\mathcal{R}_{1}, \mathcal{R}_{M+1} ; \beta\right)$,

where $Z=\int d \mathcal{R} \rho(\mathcal{R}, \mathcal{R} ; \beta)$ is the partition function. Equation (2) can be expressed by using the energy estimators proposed in Refs. 54 and 55 as

$$
\begin{aligned}
\langle E(T)\rangle= & \left\langle\frac{3(N-1)}{2} k_{B} T-\frac{1}{2 M} \sum_{\alpha=1}^{M} \sum_{i=1}^{N}\left(\mathbf{r}_{i}^{\alpha}-\mathbf{r}_{i}^{M}\right) \cdot \mathbf{F}_{i}^{\alpha}\right. \\
& \left.+\frac{1}{M} \sum_{\alpha=1}^{M} \sum_{i<j}^{N} V\left(\mathrm{r}_{i j}^{\alpha}\right)\right\rangle,
\end{aligned}
$$

where $\mathbf{r}_{i}^{M}=M^{-1} \sum_{\alpha=1}^{M} \mathbf{r}_{i}^{\alpha}$ and $\mathrm{r}_{i j}^{\alpha}=\left|\mathbf{r}_{i}^{\alpha}-\mathbf{r}_{j}^{\alpha}\right|$. The first term in Eq. (3) corresponds to the classical kinetic energy where the degrees of freedom from the center of mass (CM) have been subtracted, the second one is a quantum correction where $\mathbf{F}_{i}^{\alpha}=-\sum_{j \neq i}^{3} \nabla_{\mathbf{r}_{i}^{\alpha}} V\left(\mathrm{r}_{i j}^{\alpha}\right)$ is the force experienced by the $i$-particle on the $\alpha$ slice, and the third term describes the interaction between each pair of particles on that $\alpha$ slice. Finally, the integration is carried out via a Metropolis $\mathrm{MC}$ algorithm as an average over a number of paths $\left\{\mathcal{R}_{1}, \mathcal{R}_{2}, \ldots, \mathcal{R}_{M}, \mathcal{R}_{M+1}\right\}$ sampled according to a probability density proportional to the factorized product of $M$ density matrices of Eq. (1).

The $M$ number defines the beads which constitute each polymer or quantum path, ${ }^{52}$ and here it is taken as $M$ $=160 / T$, twice the value adopted by Borrmann, ${ }^{32}$ which we found not to be sufficient for temperatures larger than $20 \mathrm{~K}$. The specific case $M=1$ corresponds to the classical description of the system. ${ }^{32}$ A study of the convergence of the PIMC calculation is included for $T=1 \mathrm{~K}$ and $T=10 \mathrm{~K}$ in Fig. 1 . The results, even corresponding to a sole trajectory, indicate that the number of beads adopted for the PIMC calculation ensures the convergence of the calculated energies. 


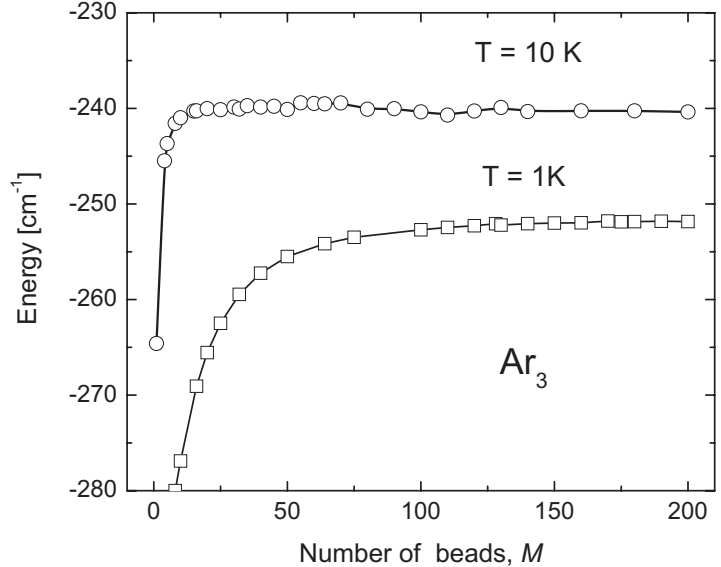

FIG. 1. Energy, in $\mathrm{cm}^{-1}$, of the $\mathrm{Ar}_{3}$ system as a function of the number of beads $M$ of the PIMC method for $T=1 \mathrm{~K}$ (squares) and $T=10 \mathrm{~K}$ (circles) for a typical trajectory.

The fluctuations in the average energy are directly related to the experimentally observable heat capacity or specific heat $C_{v}$ as follows: ${ }^{56}$

$$
\begin{aligned}
\langle E(T)\rangle^{2}-\langle E(T)\rangle^{2} & =-\left(\frac{\partial\langle E(T)\rangle}{\partial \beta}\right)=k_{B} T^{2}\left(\frac{\partial\langle E(T)\rangle}{\partial T}\right) \\
& =k_{B} T^{2} C_{v} .
\end{aligned}
$$

This relation is of general validity and applies to physical systems in which either classical or quantum effects are important. Its behavior as a function of $T$ has been commonly considered as a possible indicator of phase transitions for this kind of systems. In this work, we have calculated, according to Eq. (4), the specific heat by differentiating the average energy with respect to $T$.

The present study also includes the analysis of radial distributions of the distance between an $\mathrm{Ar}$ atom and the $\mathrm{CM}$ of the cluster calculated as

$$
\rho(\mathrm{r})=\frac{1}{6 M} \sum_{\alpha=1}^{M} \sum_{i=1}^{3} \delta\left(\mathrm{r}-\left|\mathbf{r}_{i}^{\alpha}-\mathbf{R}_{\mathrm{CM}}^{\alpha}\right|\right),
$$

where $\mathbf{R}_{\mathrm{CM}}^{\alpha}$ is the position vector for the CM in each $\alpha$ slice.

The PIMC calculations have been performed confining the Ar atoms inside a sphere centered at the $\mathrm{CM}$ with a radius $R_{c}$. As a general procedure, in order to estimate the value of a specific observable $\mathcal{A}$ at a certain temperature, between 10 and 100 iterative runs are carried out between $t_{0}$, at which the potential energy function can be considered already stabilized along the MC propagation, and up to a certain final iteration step $t_{\text {fin }}$. In each iteration of the $r$ th run, a staging multislice moves procedure ${ }^{52}$ has been applied, where a randomly chosen set of coordinates of one particle in various $\alpha$ slices is varied. Once the movement is accepted, we evaluate the values $\mathcal{A}\left(\mathcal{R}_{\alpha}\right)$ for the $M$ slices. Thus, for each iteration $t$, one can obtain the following average value:

$$
[\overline{\mathcal{A}}(t)]_{r}=M^{-1} \sum_{\alpha=1}^{M} \mathcal{A}\left(\mathcal{R}_{\alpha}^{(r)}\right) .
$$

When the whole MC propagation has finished, the average for that specific run $r$ is obtained as

$$
\overline{\mathcal{A}}_{r}=(\Delta t)^{-1} \sum_{t=t_{0}}^{t_{\text {fin }}}[\overline{\mathcal{A}}(t)]_{r},
$$

and finally, the value $\langle\mathcal{A}\rangle$ is calculated by averaging over all runs the results shown in Eq. (7).

\section{B. Rovibrational spectra based methods \\ 1. Distributed Gaussian functions method}

This method, employed to calculate the rovibrational energy levels for different values of $J$, has been described previously elsewhere. ${ }^{45,46}$ It has been applied and tested in comparison with an exact hyperspherical coordinates method on the $\mathrm{Ar}_{3}$ case for $J=0,1,6,15$, and $20{ }^{46,57}$ In essence, the procedure consists of the diagonalization of the total Hamiltonian, which is supposed to be separated in a purely vibrational part, $J=0$, and a rovibrational component, $J \geq 0$, on a basis set formed with the vibrational eigenstates and standard rotational functions $|k ; J \Omega M\rangle . \Omega$ and $M$ correspond to the $z$-component of the total angular momentum in the bodyfixed and space-fixed reference frames, respectively, and the index $k$ denotes the vibrational states $\Psi_{k}$. A total of $k_{\max }$ $=16$ vibrational states, belonging to the $A_{1}^{\prime}, A_{2}^{\prime \prime}$, and $E$ irreducible representations, have been included in our vibrational basis, thus covering an energy range up to $-182.9 \mathrm{~cm}^{-1}$ measured from the $\mathrm{Ar}_{2}+\mathrm{Ar}$ fragmentation threshold.

These $J=0$ eigenstates are calculated by means of an exact QM method which uses interparticle coordinates and DGF as basis functions. This approach was originally employed to study $\mathrm{Rg}$ trimers such as $\mathrm{He}_{3}, \mathrm{Ne}_{3}$, and $\mathrm{Ar}_{3} .{ }^{51,58-60}$ Reviews of subsequent applications for clusters formed with Rg atoms and different impurities can be found in Refs. 45 and 61. The $k$ vibrational states are expressed in terms of a basis which consists of normalized products of Gaussian functions for each $R_{i}$ pair coordinate. For the present study, a total number of 23 DGFs, separated by a $0.23 a_{0}$ distance, have been employed. The center of the first of these Gaussian functions has been located at $6.21 a_{0}$. With these functions, 12167 triangular arrangements are generated in the basis set.

The way to obtain the behavior of the average energy as a function of the temperature is based on the Boltzmann average of the rovibrational spectra calculated as explained above, ${ }^{47}$

$$
\begin{aligned}
\langle E(T)\rangle= & {\left[\sum_{J=0}^{J_{\max }} \sum_{l}(2 J+1) E_{l}^{J} e^{-\beta E_{l}^{J}}\right] } \\
& \cdot\left[\sum_{J=0}^{J_{\max }} \sum_{l}(2 J+1) e^{-\beta E_{l}^{J}}\right]^{-1},
\end{aligned}
$$

where $l$ stands for the corresponding rovibrational states which belong to the physically acceptable symmetry irreducible representation $A_{1}$ for each value of $J .^{46,62}$ The sum is extended to the value of the total angular momentum $J_{\max }$ at which convergence is ensured. For the largest value of the temperature analyzed here, $T=40 \mathrm{~K}, J_{\max }=80$ was found to be sufficient. 
TABLE I. Parameters for the Morse potentials employed to describe both short-amplitude and large-amplitude motions for the $\mathrm{Ar}_{3}$ system and the $\mathrm{Ar}_{2}$. Units of all parameters are in $\mathrm{cm}^{-1}$.

\begin{tabular}{ccccccccc}
\hline \hline$D_{A}$ & $B_{e}^{A}$ & $w_{A}$ & $D_{B}$ & $B_{e}^{B}$ & $w_{B}$ & $D_{\mathrm{Ar}_{2}}$ & $B_{e}^{\mathrm{Ar}_{2}}$ & $w_{\mathrm{Ar}_{2}}$ \\
\hline 262 & 0.5 & 20 & 183 & 0.02 & 10 & 99 & 0.05 & 27.4 \\
\hline \hline
\end{tabular}

\section{The two-Morse-potential model}

An alternative way to investigate the contribution from a more complete description, not only a limited number of states from the bound spectrum, is the use of a model based on analytically obtained energy levels. In particular, in the present study, we have employed both the discrete and continuum states from two Morse potentials to describe separately short-amplitude motions (potential A) and "softer", large-amplitude modes associated to linear isomers (potential B). Values for the corresponding parameters (see Table I) have been selected to provide merely a qualitative description of the PIMC results to gain physical insight of the dynamics as $T$ increases. The $\operatorname{Ar}_{2}(v)+\operatorname{Ar}$ continua are described by the combination of a Morse potential and a square box for the bound and continuum states, respectively. Finally, the Ar+Ar+Ar double continua states are described by means of bi- and tridimensional square boxes of size $L=10 \AA$.

Thus, the average energy can be written as

$$
\langle E\rangle=\frac{1}{Z(T)}\left\{\epsilon^{A}(T)+\epsilon^{B}(T)+\epsilon^{\mathrm{Ar}}(T)+\epsilon^{3 \mathrm{Ar}}(T)\right\},
$$

where the different contributions, above explained, are

$$
\begin{aligned}
& \epsilon^{A, B}(T)=\sum_{J}(2 J+1) E_{J}^{A, B} e^{-\beta E_{J}^{A, B}}, \\
& \epsilon^{\mathrm{Ar}_{2}(T)}=\sum_{v, j}(2 j+1) e^{-\beta E_{v, j}}\left(\frac{L}{\lambda_{T}(\mu)}\right)^{3}\left\{E_{v j}+\frac{3}{2} k_{B} T\right\}, \\
& \epsilon^{3 \operatorname{Ar}}(T)=\left(\frac{L}{\lambda_{T}(\mu)}\right)^{3}\left(\frac{L}{\lambda_{T}(m / 2)}\right)^{3} 3 k_{B} T .
\end{aligned}
$$

Equation (11) includes the so-called thermal wavelength expressed as $\lambda_{T}(\mu)=\left(2 \pi \hbar^{2} / \mu k_{B} T\right)^{1 / 2}$, where $\mu=2 m / 3$ is the reduced mass of the $\mathrm{Ar}-\mathrm{Ar}_{2}$ problem. The contribution of the total fragmentation in the three Ar atoms is accounted for Eq. (12), and it contains the thermal wavelength for the case of a diatom with a reduced mass of $m / 2, m$ being the mass of an $\mathrm{Ar}$ atom.

$Z(T)$ in Eq. (9), is the partition function, defined as the sum of the following terms:

$$
\begin{aligned}
& Z^{A, B}(T)=\sum_{J}(2 J+1) e^{-\beta E_{J}^{A, B}}, \\
& Z^{\operatorname{Ar}_{2}}(T)=\sum_{v, j}(2 j+1) e^{-\beta E_{v, j}\left(\frac{L}{\lambda_{T}(\mu)}\right)^{3},} \\
& Z^{3 \operatorname{Ar}}(T)=\left(\frac{L}{\lambda_{T}(\mu)}\right)^{3}\left(\frac{L}{\lambda_{T}(m / 2)}\right)^{3} .
\end{aligned}
$$

\section{RESULTS}

\section{A. Average energy}

Estimates of the energy for $0 \mathrm{~K} \leq T \leq 40 \mathrm{~K}$ have been obtained by means of the above discussed PIMC and DGF methods and are presented in Fig. 2. The dependence with $T$ of the present PIMC results is, however, significantly smoother than those from Ref. 47, limited there to $T \leq 1 \mathrm{~K}$, since the corresponding statistics has been noticeably improved with a larger number of runs. Energy results shown in Fig. 2 were obtained with the PIMC calculation using different values of a confining radius, $R_{c}=10,4$, and $2.6 \AA$, and are compared with the DGF energies. At $T \sim 0 \mathrm{~K}$, all calculations predict a value of the energy which is close to the vibrational ground level of $\mathrm{Ar}_{3}$ according to the potential interaction employed here, $-252.44 \mathrm{~cm}^{-1}$. No dependence on the actual value of $R_{c}$ is observed below $T \sim 15 \mathrm{~K}$. There are, however, noticeable differences at higher temperatures. Thus, the result obtained with the smallest confining radius, $R_{c}=2.6 \AA$, does not follow the raise of the rest of PIMC calculations. About $T \sim 20 \mathrm{~K}$, the slope of the energy curve for $R_{c}=10 \AA$ PIMC calculations starts to increase notably, indicating that such value for the confining radius seems not to avoid the total fragmentation of the system at $T \sim 40 \mathrm{~K}$. This behavior enables us to conclude that $10 \AA$ seems to be large enough to introduce no restriction at all to the movement of the system. In fact we have observed that results obtained with this confining radius are completely analog to those calculated under free-boundary conditions. The results

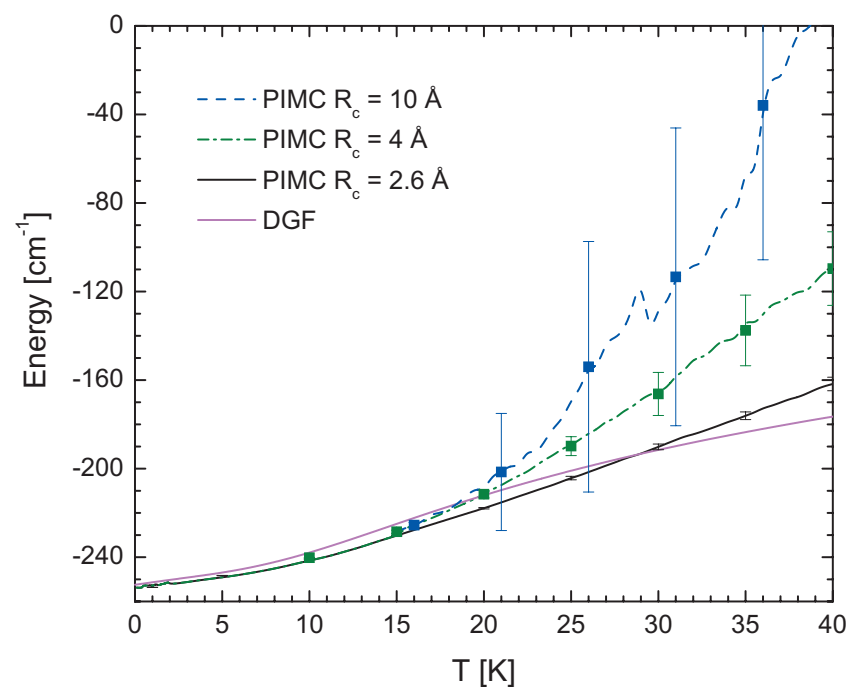

FIG. 2. Average energy, in $\mathrm{cm}^{-1}$, of the $\mathrm{Ar}_{3}$ system as a function of the temperature obtained by means of the PIMC method following Eq. (3) with values of the confining radius $R_{c}=2.6 \AA$ (black solid line), $4 \AA$ (green dashed-dotted line), $10 \AA$ (blue dashed line), and DGF according to Eq. (8) (solid magenta line) approaches. Error bars for the PIMC results have also been included. 
obtained with $R_{c}=4 \AA$, on the contrary, with much more reduced oscillations, seem to exhibit an intermediate behavior in between the previous case $R_{c}=10 \AA$ and that performed under the most limiting value for $R_{c}$ considered here, 2.6 A. The size of the error bars, also included with the PIMC results in Fig. 2, manifests, on the other hand, the noticeable difference on the stability of the results obtained with the different confining radii.

The DGF $\langle E(T)\rangle$ curve, on the other hand, displays a good overall agreement with the PIMC energies at the lower temperature regime. The moderate increase of the DGF energy is similar to the one obtained in the PIMC calculation with $R_{c}=2.6 \AA$. These two energy curves have nevertheless different slopes and cross each other at $T \sim 29 \mathrm{~K}$.

The interesting point is that the differences among the results suddenly appear around $T \sim 17-20 \mathrm{~K}$, a value which is remarkably lower than melting temperatures, $T_{m}$, reported for larger $\mathrm{Ar}$ clusters $\left(\mathrm{Ar}_{n} n \geq 13\right)$ : for $\mathrm{Ar}_{13}$ and $\mathrm{Ar}_{55}$, for example, $T_{m} \sim 40 \mathrm{~K}$ (the temperature at which, according to our PIMC results, $\mathrm{Ar}_{3}$ fragmentates into $\mathrm{Ar}+\mathrm{Ar}+\mathrm{Ar}$ ), whereas for $\mathrm{Ar}_{923} T_{m}$ has been reported to be around $68 \mathrm{~K} .^{63}$ According to our results, $\mathrm{Ar}_{3}$ has already dissociated before reaching any of these two temperatures. Below $T$ $\sim 17-20 \mathrm{~K}$, all PIMC calculations predict nonoscillating $\langle E(T)\rangle$ curves. This feature is consistent with previous findings which would indicate a distinct behavior for the three particle clusters in comparison with larger systems. ${ }^{5-7,64,65}$ The onset of abrupt changes in the energy as the temperature increases has been commonly associated with either phase transitions or significant changes in the extension of configuration space explored by the cluster. In this sense, the application of confining schemes in the PIMC approach and the absence of an erratic dependence of the energy with $T$ seem to be correlated. The results reported in the PIMC study of the $\mathrm{Ar}_{13}$ by Tsai and Jordan ${ }^{66}$ also exhibit a marked dependence on the radius of a constraining sphere centered at the $\mathrm{CM}$ of the system.

For a further investigation on this issue, we have applied the analytical model of Sec. II B 2. In Fig. 3 we have included $\langle E(T)\rangle$ values obtained for four different cases: (i) only the discrete bound states from Morse A are considered; (ii) the discrete spectra from both Morse potentials are included in the calculation; (iii) in addition to the previous case, the continuum emerging from the evaporation of one Ar atom is included and (iv) the total fragmentation continuum is also taken into account. Despite its qualitative nature, the comparison presented in Fig. 3 provides a rationale regarding the behavior of the energy in terms of the temperature. The calculation restricted to the energy levels of the bound states of the Morse A potential produces analog results to those obtained with both the PIMC calculation within the most restrictive confining radius and the DGF method. The inclusion of the bound state spectrum of the Morse B potential, conceived to describe more extended geometries explored by the system, leads to an increase of the energy curve at a certain value of the temperature. This case seems, therefore, to be correlated with the PIMC results obtained with $R_{c}=4 \AA$. But, it is only when the continua are also allowed to contribute when a pronounced raise with $T$ is found in the

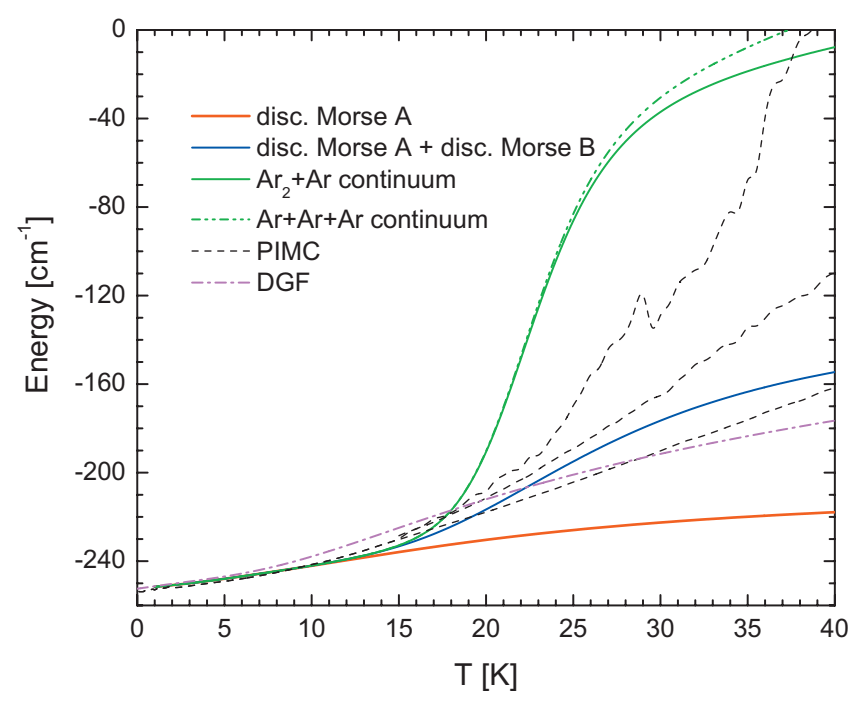

FIG. 3. Values of the average energy in terms of the temperature obtained by the model of Sec. II B 2 (with the discrete spectrum of the Morse potential A (red solid line) with the discrete spectra of both Morse potentials A and B (blue solid line) and including both the atom-diatom continuum (green solid line) and total-fragmentation continuum (green dashed-dotteddotted line). The DGF (magenta solid-dotted line) and the three different PIMC results (black dashed lines) shown in Fig. 2 have also been included for comparison.

average energy. The comparison with the $R_{c}=10 \AA$ PIMC calculation allows us to conclude that the ultimate reason for the observed behavior in terms of the temperature is the evaporation of at least one Ar atom from the cluster.

The agreement found with the PIMC results up to $T$ $\sim 20 \mathrm{~K}$ indicates that at that temperature range the dynamics of the system is governed by short-amplitude fluctuations from equilateral geometries. The observed divergence at larger values of $T$, on the other hand, has to be interpreted as a consequence of a noncomplete description of the rovibrational spectra employed in Eq. (8). More regarding the specific geometry of these apparently missing rovibrational states will be discussed in Sec. III C.

\section{B. Specific heat}

In this work we have calculated values of $C_{v}$ following the expression of Eq. (4). In order to avoid spurious oscillations in the results, the corresponding energy curves were fitted to a multipeak Gaussian function and derivatives were obtained. The so obtained $C_{v}(T)$ curves are shown in Fig. 4. Final results were found not to depend on the explicit functional form used for the interpolation fit of the energy curves, with multipeak Lorentzian or cubic spline fits yielding to essentially the same specific heats shown in Fig. 4. This procedure differs slightly from the calculation by Chakravarty ${ }^{37}$ of neon clusters, in which the fit was actually performed for the data points obtained in the simulation of the specific heat.

The three PIMC results shown in Fig. 4 exhibit, on the other hand, a distinctive behavior. Thus, the $C_{v}(T)$ curve obtained with $R_{c}=2.6 \AA$ is characterized by a broad plateau which covers the $T=20-40 \mathrm{~K}$ range. The result corresponding to the PIMC calculation when the confining radius is $4 \AA$ exhibits a peak between $T=30-35 \mathrm{~K}$, the position of which 


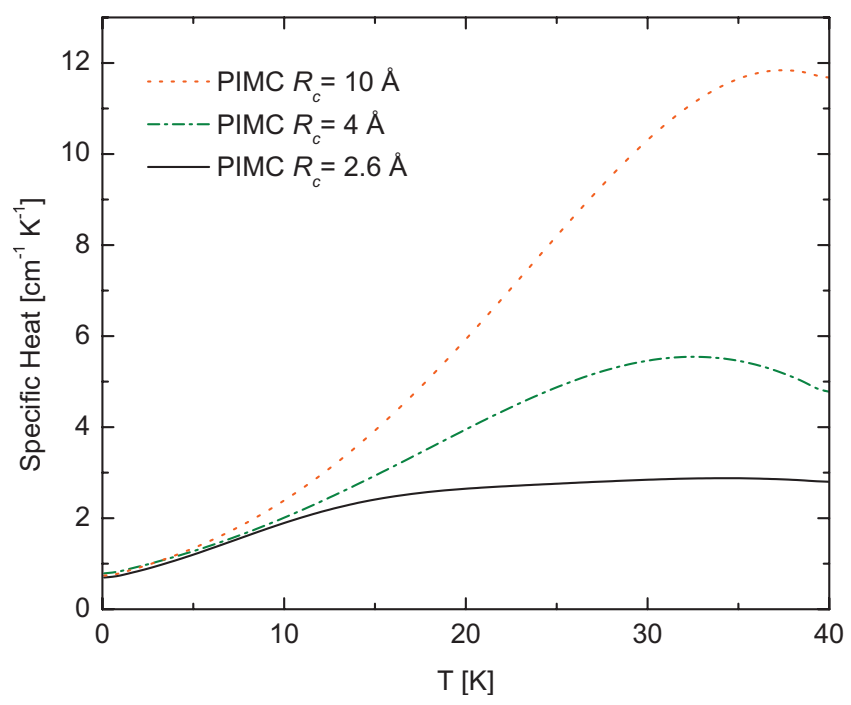

FIG. 4. Specific heats as a function of the temperature calculated with the PIMC method with $R_{c}=2.6 \AA$ (black solid line), $R_{c}=4 \AA$ (green dasheddotted line), and for $R_{c}=10 \AA$ (red dotted line).

seems to be shifted to larger temperatures for the other case investigated here, the PIMC specific heat calculated with the least restrictive case $R_{c}=10 \AA$.

The evolution of this apparent maximum with the value of the constraining radius is analog to the situation reported before for larger systems. ${ }^{66-68}$ In those previous studies, besides a prominent maximum peak whose structure does not change in any case, some other broader features which depend on the precise value of the radius for the constraining sphere employed in the simulations were observed. For the case of $\mathrm{Ar}_{13}$ (Ref. 66) this such secondary peak was attributed to evaporation effects whereas Mandelshtam and coworkers interpreted this feature for the $(\mathrm{LJ})_{n}$ clusters as indications of melting of the cluster core. ${ }^{67,68}$

Whereas the observed structureless heat capacity for $R_{c}$ $=2.6 \AA$ seems to be indicative of too restrictive limitations on the system, the results obtained here for $R_{c}=4 \AA$ are qualitatively similar to previously reported results. ${ }^{27,69}$ The absence for $\mathrm{Ar}_{3}$ of an unambiguous maximum peak with the same characteristics as those observed at $T \sim 35 \mathrm{~K}$ for the $\mathrm{Ar}_{13}$ case $^{66}$ or at the low-temperature regime for large $(\mathrm{LJ})_{n}$ clusters $^{67,68}$ could be consistent with the occurrence of structural changes or isomerization ${ }^{69}$ rather than a proper phase transition as observed for larger clusters. The almost simultaneous shift to different geometrical arrangements should not be discarded either. We would discuss about the existence of such structures in Sec. III B.

\section{Geometrical structure}

The apparent independence of the PIMC results at the low temperature regime $(T \leq 15 \mathrm{~K})$ with respect to the precise value of the confining radius could be interpreted as a consequence of small fluctuations of the system around rigid spatial arrangements. The most likely average geometry explored by $\mathrm{Ar}_{3}$ at such low temperature should be the equilibrium equilateral structure. This scenario is nevertheless found to change dramatically as the temperature reaches

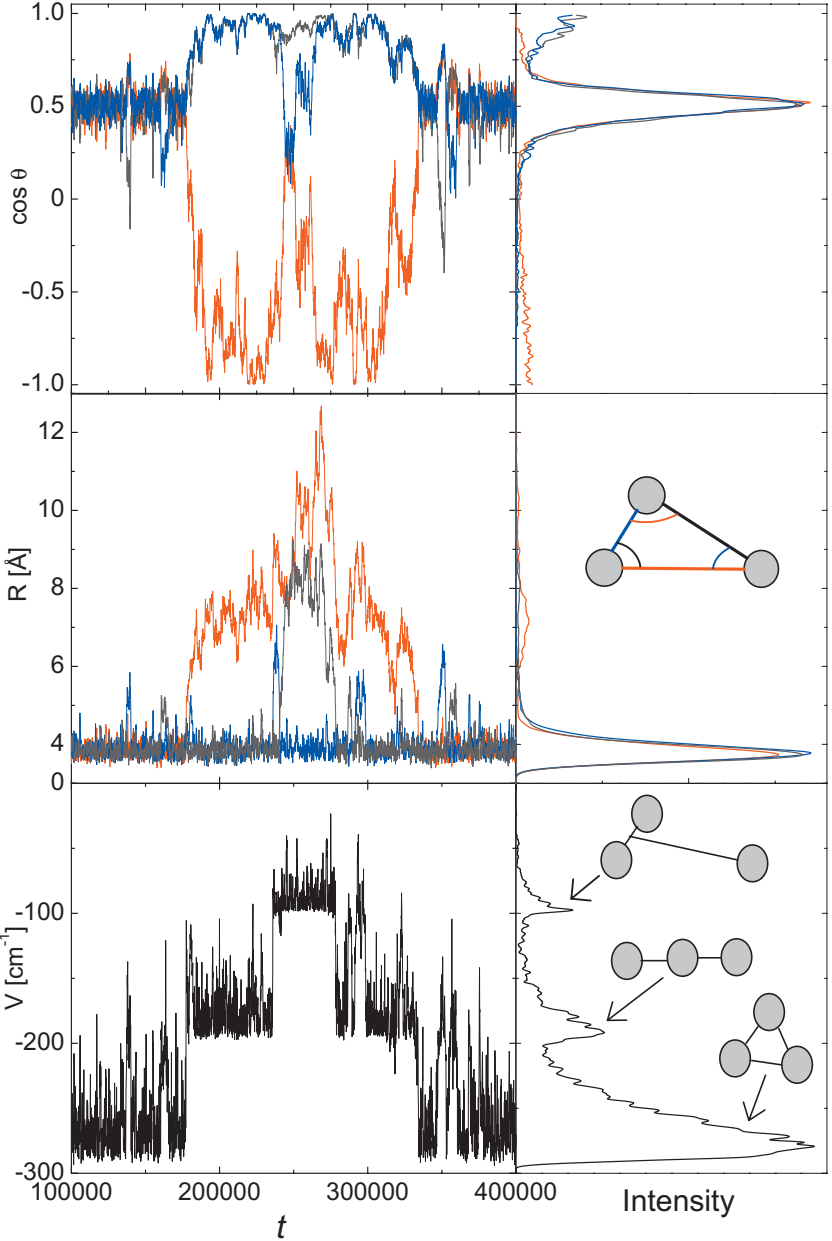

FIG. 5. (Bottom left panel) PIMC values for the potential energy, in $\mathrm{cm}^{-1}$, in terms of $t$ for a generic run at $T=22 \mathrm{~K}$; (middle left panel) Interparticle distance, $R$, in angstrom. Red/black/blue are for the largest/intermediate/ smallest distances, respectively; (top left panel) Values of the three cosines. Colors depend on the opposite side of the triangle. Histograms with the cumulative intensities, in arbitrary units, for the potential energy (bottom right panel), distances (middle right panel), and cosines (top right panel) are also shown. Geometries explored by the $\mathrm{Ar}_{3}$ system for each average value of the potential energy (bottom right panel) and the scheme with colors for both distances and cosines are also included (middle right panel).

$20 \mathrm{~K}$. Beyond this specific value of $T$, the PIMC runs for $R_{c}=10 \AA$ describe how the system starts to explore substantially different geometries.

One can gain an illustrative idea of the apparent freedom of the cluster by inspecting the values of the potential energy $V$ as a function of the PIMC iteration step for a representative run at a sufficiently large temperature, $T=22 \mathrm{~K}$. The analysis of $V$ shown in Fig. 5 will enable us to establish a rationale in terms of specific configurations adopted by the trimer. Similar analysis have been reported before for this sort of systems. ${ }^{27,70,71}$

One of the main characteristics of results shown in Fig. 5 is the highly oscillating behavior of $V$ as $t$ increases. Despite these oscillations, three different stability regions for the average potential energies can be appreciated around $\sim-264 \mathrm{~cm}^{-1}, \sim-179 \mathrm{~cm}^{-1}$, and $\sim-90 \mathrm{~cm}^{-1}$. The corresponding histograms in the right side panels show peaks at these particular values. It is possible to relate these energies with specific arrangements of the three Ar atoms. Thus, the 
lowest value, $V \sim-264 \mathrm{~cm}^{-1}$, could correspond to the potential energy for the ground state of the Ar trimer, $-252.4 \mathrm{~cm}^{-1}$, which is preferentially found in an equilateral triangle geometry. In turn, the least bound situation, $V \sim-90 \mathrm{~cm}^{-1}$, seems not to differ from either the energy of the ground state of the Ar dimer (which, for the potential energy function employed here is $-83.94 \mathrm{~cm}^{-1}$ ) or the bottom of the $\mathrm{Ar}-\mathrm{Ar}$ potential, $D=99 \mathrm{~cm}^{-1}$. The system would be then close to a fragmentation or evaporation at this stage of the propagation which would yield $\mathrm{Ar}_{2}+\mathrm{Ar}$. The intermediate energy, $V \sim-179 \mathrm{~cm}^{-1}$, can be understood as roughly the addition of the energies of two separate $\mathrm{Ar}-\mathrm{Ar}$ bonds, which strictly leads to $-167.88 \mathrm{~cm}^{-1}$, or even to twice the value of $D$. This situation is fulfilled for linear geometries where the Ar atom located at the center seems to screen the possible interaction between the two other atoms. In fact, for the Morse potential used in this work, indications of a barrier for linearity have been found for an energy of about $-168 \mathrm{~cm}^{-1} .59,72$ The energy level of the last $\mathrm{Ar}_{3}$ vibrational state $\left(k_{\max }=16\right)$ in our basis is below such a barrier and therefore linear structures are excluded in our DGF calculation. The passage from the nearly rigid equilateral triangle form to the nonrigid linear structure was also reported in the MD study on $\mathrm{Ar}_{3}$ by Beck et al., ${ }^{21}$ and it was interpreted as indicative of internal mode softening of the system. Schemes of the configurations that the system seems to be exploring during the PIMC propagation have been included in Fig. 5.

The above discussed energy considerations can be completed with similar plots for geometrical quantities. In particular, Fig. 5 includes the behavior of the distances and cosines of the corresponding triangular structures displayed by the system in the same run. Our preliminary conclusions extracted from such analysis are corroborated by the inspection of results shown in Fig. 5. Thus, those regions which were attributed to equilateral geometries correspond to equal values $\left(\sim 3.7 \AA\right.$ ) of $R_{1}, R_{2}$, and $R_{3}$, and the three corresponding angles $\theta_{i} \sim 60^{\circ}$; those ascribed to linear geometries have $R_{1}$, $R_{2} \sim 3.7 \AA, R_{3} \sim 7 \AA$ and angles $\theta_{1,2} \sim 0^{\circ}, \theta_{3} \sim 180^{\circ}$, and finally, for the values of the PIMC simulation step between $t \sim 230000$ and 280000 , at least one of the three interparticle distances can take values approximately equal to $12 \AA$, a sufficiently large enough value to consider that one of the Ar atoms is close to be lost in a cluster fragmentation process. The analysis of the corresponding histograms, shown at the right side panels of Fig. 5, exhibits marked maxima at $R \sim 3.7 \AA$ and $\cos \theta \sim 0.5$ for the pair distance and cosine distributions, respectively. Much less pronounced are the intensities for the maxima suggested at $R \sim 7 \AA$ and $\cos \theta$ $\sim 1$ and -1 .

Both PIMC and MD simulations have previously revealed evidences of frequent transitions between stable isomers for $\operatorname{Rg}_{n}(n \leq 19)$ clusters. ${ }^{5,20,35,73,74}$ In particular, as an example of a three-particle system, the monitored interatomic distances in the MD calculations on $\mathrm{Ar}_{3}^{+}$performed by Calvo et al. $^{25}$ also revealed the transition between two permutational isomers of the ground state geometry (which is linear for this ionic cluster) with the presence of a loosely bound intermediate minimum (T-shaped for this system).

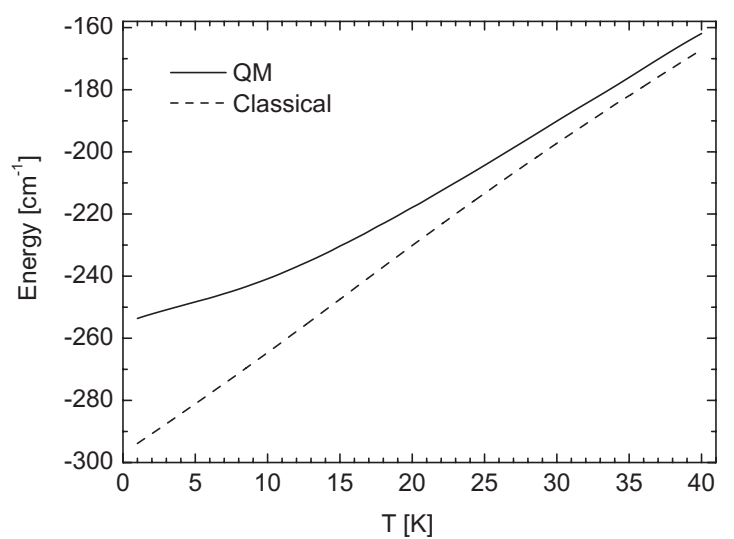

FIG. 6. Average energy of the $\mathrm{Ar}_{3}$ system as a function of the temperature calculated with the QM (solid line) and classical version (dashed line) of the PIMC method with $R_{c}=2.6 \AA$.

\section{Classical versus quantum mechanical description}

Quantum and classical values for the energy obtained with the PIMC method have been compared as a function of the temperature. In particular, in Fig. 6 we show the results for the case of $R_{c}=2.6 \AA$, already displayed in Fig. 2, in comparison with the corresponding classical counterpart.

The differences between both sets of data are evident at the lowest temperature range, specially for $T=1 \mathrm{~K}$, with the QM energy about $40 \mathrm{~cm}^{-1}$ larger than the classical values. The almost linearly increasing trend of the classical energy seems, however, to be followed by the QM $\langle E(T)\rangle$ curve beyond $T \geq 20 \mathrm{~K}$. Results in Fig. 6 resemble those obtained for $\mathrm{Ar}_{6}$ (Ref. 30) and $\mathrm{Ar}_{13}{ }^{30,32}$ Classical energies, especially at the lowest temperatures, remain clearly below the QM values. Interestingly the results reported in Ref. 30 indicate that the sudden increases in the $\langle E(T)\rangle$ curves, due exclusively to the potential energy part, occur in both the classical and QM calculations. This is not the case for $\mathrm{Ar}_{3}$, since no abrupt change in the dependence of the energy is found in the classical result.

Pair correlation functions for Ar clusters obtained by classical approaches have been found to differ significantly from those calculated quantum mechanically at low temperature. In this work, probability density functions for the distance of an Ar atom to the $\mathrm{CM}$ of the system $\left(R_{\mathrm{CM}}\right)$ with both the classical and QM PIMC at $T=1,5$, and $15 \mathrm{~K}$ have been calculated. $R_{c}$ has been increased slightly to $3 \AA$ to avoid excessively compressed distributions. The comparison between both kinds of radial functions is shown in Fig. 7. The dependence with the temperature is markedly more pronounced for the classical calculation than for the QM version. Thus, the classical radial density, which at $T=1 \mathrm{~K}$ is narrow and peaked about $\sim 2.2 \AA$, becomes much broader and with a smaller maximum peak as the temperature is increased up to 5 and $15 \mathrm{~K}$. Furthermore, the distribution, and more particularly the location of the maximum, shifts to slightly larger values of $R_{\mathrm{CM}}$. On the contrary, the QM distributions at $T=1 \mathrm{~K}$ and $T=5 \mathrm{~K}$ are almost the same, much less pronounced than their corresponding classical counterparts. The only appreciable change appears when the temperature becomes $15 \mathrm{~K}$. The radial density function becomes 


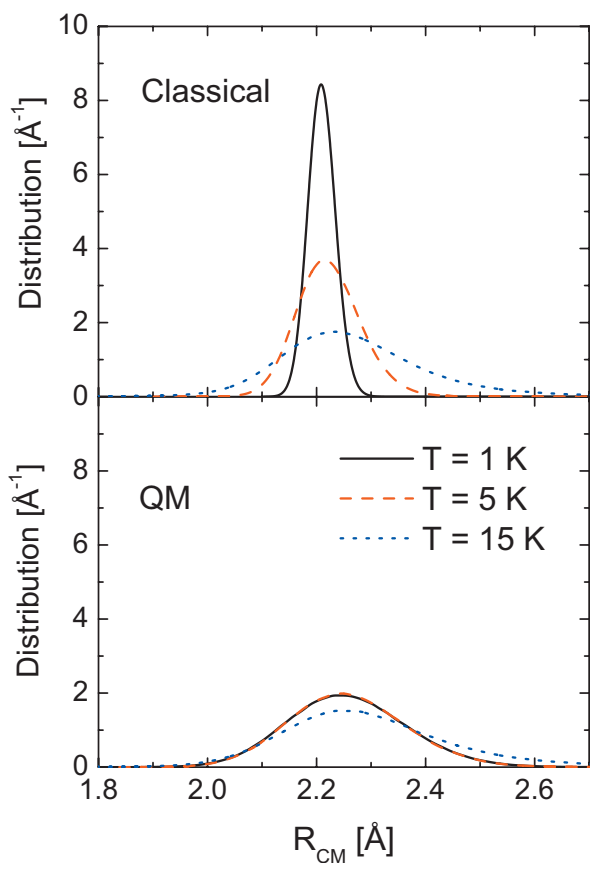

FIG. 7. Radial probability density functions in terms of the distance of any of the three Ar atoms to the $\mathrm{CM}$ of the trimer obtained with the PIMC with $R_{c}=3 \AA$ in its classical $(M=1)$ (top panel) and $\mathrm{QM}$ versions (bottom panel) at $T=1 \mathrm{~K}$ (black solid line), $5 \mathrm{~K}$ (red dashed line), and $15 \mathrm{~K}$ (blue dotted line).

smaller and, as in the classical case, begins to develop a propensity to extend to further distances from the $\mathrm{CM}$ of the system. This feature is consistent with the apparent major mobility that the cluster exhibits when the temperature gets close to about $20 \mathrm{~K}$, as we have discussed in Secs. III A and III C. Interestingly, according to Fig. 6, T=15 K corresponds to a temperature at which the zero-energy point seems to have been already reached.

The broadening of the pair correlation functions with the temperature has been well documented in previous studies of larger Rg clusters, $6,730,32,37,64,65$ and is commonly interpreted as indicative of structural transitions or phase transitions experimented by the system. In addition, much broader distributions have been also observed in the QM calculations than in the classical ones at low temperatures for the cases of $\mathrm{Ar}_{6},{ }^{30} \mathrm{Ar}_{13},{ }^{32}$ or $\mathrm{Ne}_{13}{ }^{37}$ Those differences, as in the present case of the Ar trimer, were found to diminish as the temperature increases. For $\mathrm{Ne}_{38}$, for example, the radial pair correlation functions calculated with PIMC, as opposed to the result found for classical simulations, also exhibit no abrupt change in the temperature interval $2 \mathrm{~K} \leq T \leq 10 \mathrm{~K}^{41}$

\section{DISCUSSION}

Since both the linear and the atom-diatom arrangements are available for the system when the confinement restrictions are relaxed, it is interesting to wonder about the best manner to calculate average values for relevant magnitudes when the system exhibits such a variety of possible distinct geometries. In view of the situation shown in Fig. 5 one would think that the correct thing to do would be to average separately over the different regions of stability. In fact, this procedure has been adopted in the past in different occasions.
Thus, previous studies have focused on the separate contribution from the different stable isomers of Ar clusters or even more specifically from the solidlike and liquidlike forms. ${ }^{22,35,73,74}$ In particular, values of the average energy, ${ }^{64}$ velocity autocorrelation functions, ${ }^{74}$ and mean square displacements ${ }^{74}$ over segments formed by selected portions of trajectories or runs attributed to specific forms or phases have been reported.

Whether or not this higher degree of mobility which appears beyond $T \sim 20 \mathrm{~K}$ is indicative of a phase transition for such a small cluster is an interesting issue to discuss. Different authors have preferred to interpret these features in terms of passages over saddle points to access existing stable isomers located in potential energy minima. ${ }^{20,21}$ The motion between the competing structures in $\mathrm{Ar}_{13}$, for instance, was considered complex enough to be considered as liquidlike. ${ }^{30}$ Furthermore, the possible absence of those stable forms in the case of the smallest $\operatorname{Ar}_{n}$ microclusters $(n<7)$ has been suggested to be at the origin of phase-transition behavior only for clusters with more than seven Ar atoms. ${ }^{5,31}$ Conformational transitions within $\mathrm{Rg}_{4}$ clusters were also connected with the melting of these systems by Elyutin et al. ${ }^{27}$ In line with these interpretations for this sort of phenomena, and in view of the present results, what one could conclude is that at sufficiently high temperatures the Ar trimer is capable to explore more spatially delocalized structures, thus abandoning the rigid equilateral arrangement which seems to dominate the overall geometry at the low temperature regime. Therefore, despite the abrupt change in the temperature dependence of the energy resembles some features found for larger clusters, the observed behavior of the specific heats indicates that this dynamical evolution does not constitute however a proper phase transition. This would then question the report of a specific value for a liquid-gas transition as done in Ref. 6. Recent findings for this system also support this conclusion. ${ }^{28}$

It is worth pointing out that the here proposed methods to investigate the average energy in terms of $T$ constitute, in a sense, a noticeable improvement of previous comparisons between zero temperature MC approaches and the PIMC method. Rick et al., for example, compared estimates of the ground-state energies obtained by means of diffusion MC calculations with the limiting low-temperature values calculated with a Fourier PIMC approach. ${ }^{35}$ The scheme based on the Boltzmann average of Eq. (8), which has been used in this work, enables us, on the contrary, to extend this sort of comparison to a reasonably larger interval of temperatures.

The multinormal-modes (MNM) model by Franke et $a l .{ }^{75}$ probably constitutes a somehow analogous alternative to the PIMC as the present DGF approach. In that model, the stable isomers of the cluster are initially identified, assigning an equal amount of phase-space-volume to each one. The equilibrium distribution is calculated with respect to the energies of those isomers. Thus, instead of rovibrational state energies, eigenfrequencies of the stable isomers are employed. In some way, the MNM model is therefore strongly connected with those interpretations of the observed changes in the thermal properties of Ar microclusters in terms exclusively of the existing stable forms of such systems. Interest- 
ingly, analogous deviations with respect to the corresponding PIMC calculation, as shown here, have been found in the analysis of the $\mathrm{Ar}_{6}$ and $\mathrm{Ar}_{13}$ clusters by means of this MNM approach. Thus, beyond a certain value of the temperature ( $T \sim 10 \mathrm{~K}$ and $T \sim 30 \mathrm{~K}$ for $\mathrm{Ar}_{6}$ and $\mathrm{Ar}_{13}$, respectively) the MNM energies clearly underestimate the thermal energy calculated with the PIMC approach. For $\mathrm{Ar}_{6}$ both the PIMC and MNM energy curves were found to differ at that temperature at which the best-single cluster approximation (that only takes into account the contribution from the absoluteminimum configuration) is probably not good enough. That led the authors of Ref. 75 to attribute the failure of the MNM model to a deficient description of the existing isomerization experienced by the cluster.

\section{CONCLUSIONS}

The present work is an exhaustive review on the thermal properties of the $\mathrm{Ar}_{3}$ system. Average energies, specific heats, and radial distributions have been calculated by means of a PIMC approach and an approximate method based on the Boltzmann average of the rovibrational energies at sufficiently large values of the total angular momentum. The calculation of the corresponding rovibrational spectra is carried out with a recently proposed method which employs DGFs and pair coordinates. The comparison of energies obtained by these two approaches reveals a good agreement for temperatures which go up to $T \sim 20 \mathrm{~K}$. Beyond that temperature, the DGF method, which employs a reduced vibrational basis with no bound states beyond the barrier for linearity, underestimates the PIMC energies.

For the high temperature regime, $T>20 \mathrm{~K}$, the Ar trimer is found to explore more delocalized geometrical configurations. As a result, the energy begins to manifest an erratic behavior as a function of the temperature which is only controlled by using a restrictive confining radius with the PIMC method. Interestingly, as we relax the restrictions imposed on the system spatially more extended configurations, such as linear geometries or arrangements in which one Ar atom is located further away from the remaining $\mathrm{Ar}_{2}$ dimer, are explored by the system. An analytical model has been used for a rationale of the dynamics of the trimer with a precise account for the role played by the dissociative continuum, which has been generally omitted in previous studies. The present findings thus reveal that the Ar trimer exhibits the onset of profound alterations in its dynamics at a much lower temperature that larger $\operatorname{Ar}_{n}(n \gg 3)$ systems and in fact, the melting temperature reported for such systems, are usually well above the total dissociation temperature found here: $T \sim 40 \mathrm{~K}$.

A careful analysis of the specific heat in terms of the constraining radius reveals that the Ar trimer seems not to experience a proper phase transition. The absence of unambiguous peaks as a function of the temperature is, on the contrary, an indication of the occurrence of mere structural changes. This conclusion will then question previous reports of specific values for an apparent liquid-gas transition of this trimer.

QM effects are evident, specially at low temperatures
$T<15-20 \mathrm{~K}$, with notable differences between classical and quantum PIMC results for both energies and radial densities. These findings confirm previous results for larger Ar clusters. For the present case, this study provides the value for the specific temperature range at which classical approaches are expected to constitute valid methods to treat the title system

\section{ACKNOWLEDGMENTS}

This work has been funded by the MEC (Spain) under Project Nos. FIS2007-62006 and CTQ2007-62898. We would like to thank Professor Julius Jellinek for fruitful and interesting discussions. The calculations have been performed at Centro de Cálculo (IFF), CTI (CSIC), and Cesga (the Supercomputer Center of Galicia).

${ }^{1}$ R. S. Berry, T. L. Beck, H. L. Davis, and J. Jellinek, Adv. Chem. Phys. 70, 75 (1988)

${ }^{2}$ J. Dunkel and S. Hilbert, Physica A 370, 390 (2006).

${ }^{3}$ D. J. McGinty, J. Chem. Phys. 58, 4733 (1973).

${ }^{4}$ W. Kristensen, E. Jensen, and R. Cotteril, J. Chem. Phys. 60, 4161 (1974).

${ }^{5}$ C. L. Briant and J. J. Burton, J. Chem. Phys. 63, 2045 (1975).

${ }^{6}$ R. D. Etters and J. Kaelberer, Phys. Rev. A 11, 1068 (1975).

${ }^{7}$ R. D. Etters and J. Kaelberer, J. Chem. Phys. 66, 5112 (1977).

${ }^{8}$ R. P. White, S. M. Cleary, and H. R. Mayne, J. Chem. Phys. 123, 094505 (2005).

${ }^{9}$ A. J. Stace, Chem. Phys. Lett. 99, 470 (1983).

${ }^{10}$ T. E. Gough, D. G. Knight, and G. Scoles, Chem. Phys. Lett. 97, 155 (1983).

${ }^{11}$ D. Eichenauer and R. J. Le Roy, Phys. Rev. Lett. 57, 2920 (1986).

${ }^{12}$ D. Eichenauer and R. J. Le Roy, J. Chem. Phys. 88, 2898 (1988).

${ }^{13}$ J. Bösiger and S. Leutwyler, Phys. Rev. Lett. 59, 1895 (1987).

${ }^{14}$ M. Y. Hahn and R. L. Whetten, Phys. Rev. Lett. 61, 1190 (1988).

${ }^{15}$ J. Bösiger, R. Knochenmuss, and S. Leutwyler, Phys. Rev. Lett. 62, 3058 (1989).

${ }^{16}$ J. E. Adams and R. M. Stratt, J. Chem. Phys. 93, 1358 (1990).

${ }^{17}$ L. E. Fried and S. Mukamel, Phys. Rev. Lett. 66, 2340 (1991).

${ }^{18}$ M. Schmidt, J. L. Calve, and M. Mons, J. Chem. Phys. 98, 6102 (1993).

${ }^{19}$ F. G. Amar and R. S. Berry, J. Chem. Phys. 85, 5943 (1986).

${ }^{20}$ T. L. Beck, J. Jellinek, and R. S. Berry, J. Chem. Phys. 87, 545 (1987).

${ }^{21}$ T. L. Beck, D. M. Leitner, and R. S. Berry, J. Chem. Phys. 89, 1681 (1988).

${ }^{22}$ G. Natanson, F. Amar, and R. S. Berry, J. Chem. Phys. 78, 399 (1983).

${ }^{23}$ I. Benjamin, Y. Alhassid, and R. D. Levine, Chem. Phys. Lett. 115, 113 (1985).

${ }^{24}$ R. Kalus, D. Hrivnak, and A. Vitek, Chem. Phys. 325, 278 (2006).

${ }^{25}$ F. Calvo, F. X. Gadea, A. Lombardi, and V. Aquilanti, J. Chem. Phys. 125, 114307 (2006).

${ }^{26}$ D. M. Leitner, J. D. Doll, and R. M. Whitnell, J. Chem. Phys. 94, 6644 (1991).

${ }^{27}$ P. Elyutin, V. Baranov, E. Belega, and D. Trubnikov, J. Chem. Phys. 100, 3843 (1994)

${ }^{28}$ V. Aquilanti, A. Lombardi, M. B. Sevryuk, and E. Yurtsever, Phys. Rev. Lett. 93, 113402 (2004).

${ }^{29}$ D. Leitner, R. Berry, and R. Whitnell, J. Chem. Phys. 91, 3470 (1989).

${ }^{30}$ G. Franke, E. R. Hilf, and L. Polley, Z. Phys. D: At., Mol. Clusters 9, 343 (1988).

${ }^{31}$ G. Franke and J. Schulte, Z. Phys. D: At., Mol. Clusters 12, 65 (1989).

${ }^{32}$ P. Borrmann, Comput. Mater. Sci. 2, 593 (1994).

${ }^{33}$ T. L. Beck, J. D. Doll, and D. L. Freeman, J. Chem. Phys. 90, 5651 (1989).

${ }^{34}$ D. D. Frantz, D. L. Freeman, and J. D. Doll, J. Chem. Phys. 97, 5713 (1992).

${ }^{35}$ S. W. Rick, D. L. Leitner, J. D. Doll, D. L. Freeman, and D. D. Frantz, J. Chem. Phys. 95, 6658 (1991).

${ }^{36}$ D. D. Frantz, D. L. Freeman, and J. D. Doll, J. Chem. Phys. 93, 2769 (1990).

${ }^{37}$ C. Chakravarty, J. Chem. Phys. 102, 956 (1995).

${ }^{38}$ G. E. López, J. Chem. Phys. 117, 2225 (2002). 
${ }^{39}$ C. Predescu, D. Sabo, J. D. Doll, and D. L. Freeman, J. Chem. Phys. 119, 12119 (2003).

${ }^{40}$ D. Sabo, C. Predescu, J. D. Doll, and D. L. Freeman, J. Chem. Phys. 121, 856 (2004).

${ }^{41}$ C. Predescu, P. A. Frantsuzov, and V. A. Mandelshtam, J. Chem. Phys. 122, 154305 (2005).

${ }^{42}$ P. A. Frantsuzov, D. Meluzzi, and V. A. Mandelshtam, Phys. Rev. Lett. 96, 113401 (2006).

${ }^{43}$ J. Deckman, P. A. Frantsuzov, and V. A. Mandelshtam, Phys. Rev. E 77, 052102 (2008).

${ }^{44}$ J. Deckman and V. A. Mandelshtam, Phys. Rev. E 79, 022101 (2009).

${ }^{45}$ I. Baccarelli, F. A. Gianturco, T. González-Lezana, G. Delgado-Barrio, S. Miret-Artés, and P. Villarreal, Phys. Rep. 452, 1 (2007).

${ }^{46}$ M. Márquez-Mijares, T. González-Lezana, O. Roncero, S. Miret-Artés, G. Delgado-Barrio, and P. Villarreal, Chem. Phys. Lett. 460, 417 (2008).

${ }^{47}$ R. Pérez de Tudela, M. Márquez-Mijares, T. González-Lezana, O. Roncero, S. Miret-Artés, G. Delgado-Barrio, and P. Villarreal, Few-Body Syst. 45, 237 (2009).

${ }^{48}$ P. V. Elutin, V. I. Baranov, E. D. Belega, and D. N. Trubnikov, Chem. Phys. Lett. 196, 1 (1992).

${ }^{49}$ D. L. Freeman and J. D. Doll, J. Chem. Phys. 82, 462 (1985).

${ }^{50}$ F. Calvo, J. P. K. Doye, and D. J. Wales, J. Chem. Phys. 114, 7312 (2001).

${ }^{51}$ T. González-Lezana, J. Rubayo-Soneira, S. Miret-Artés, F. A. Gianturco, G. Delgado-Barrio, and P. Villarreal, J. Chem. Phys. 110, 9000 (1999).

${ }^{52}$ D. M. Ceperley, Rev. Mod. Phys. 67, 279 (1995).

${ }^{53}$ Y. Kwon, D. M. Ceperley, and K. B. Whaley, J. Chem. Phys. 104, 2341 (1996).

${ }^{54}$ M. F. Herman, E. J. Bruskin, and B. J. Berne, J. Chem. Phys. 76, 5150 (1982).

${ }^{55}$ K. R. Glaesemann and L. E. Fried, J. Chem. Phys. 116, 5951 (2002).

${ }^{56}$ R. K. Pathria, Statistical Mechanics, 2nd ed. (Butterworth, Oxford, 1996).
${ }^{57}$ M. Márquez-Mijares, R. Pérez de Tudela, T. González-Lezana, O. Roncero, S. Miret-Artes, G. Delgado-Barrio, P. Villarreal, I. Baccarelli, F. A. Gianturco, and J. Rubayo-Soneira, J. Chem. Phys. 130, 154301 (2009).

${ }^{58}$ T. González-Lezana, J. Rubayo-Soneira, S. Miret-Artés, F. A. Gianturco, G. Delgado-Barrio, and P. Villarreal, Phys. Rev. Lett. 82, 1648 (1999).

${ }^{59}$ I. Baccarelli, F. A. Gianturco, T. González-Lezana, G. Delgado-Barrio, S. Miret-Artés, and P. Villarreal, J. Chem. Phys. 122, 144319 (2005).

${ }^{60}$ I. Baccarelli, F. A. Gianturco, T. González-Lezana, G. Delgado-Barrio, S. Miret-Artés, and P. Villarreal, J. Chem. Phys. 122, 084313 (2005).

${ }^{61}$ T. González-Lezana, S. Miret-Artés, G. Delgado-Barrio, P. Villarreal, J. Rubayo-Soneira, I. Baccarelli, F. Paesani, and F. A. Gianturco, Comput. Phys. Commun. 145, 156 (2002)

${ }^{62}$ F. Karlický, B. Lepetit, R. Kalus, and F. X. Gadéa, J. Chem. Phys. 126, 174305 (2007).

${ }^{63}$ E. Pahl, F. Calvo, L. Koçi, and P. Schwerdtfeger, Angew. Chem., Int. Ed. 47, 8207 (2008).

${ }^{64}$ R. D. Etters, R. Danilowicz, and J. Kaelberer, J. Chem. Phys. 67, 4145 (1977).

${ }^{65}$ J. B. Kaelberer and R. D. Etters, J. Chem. Phys. 88, 7 (1977).

${ }^{66}$ C. J. Tsai and K. D. Jordan, J. Chem. Phys. 99, 6957 (1993).

${ }^{67}$ P. A. Frantsuzov and V. A. Mandelshtam, Phys. Rev. E 72, 037102 (2005).

${ }^{68}$ V. A. Mandelshtam and P. A. Frantsuzov, J. Chem. Phys. 124, 204511 (2006).

${ }^{69}$ E. Yurtsever and F. Calvo, Phys. Rev. B 62, 9977 (2000).

${ }^{70}$ H. L. Davis, J. Jellinek, and R. S. Berry, J. Chem. Phys. 86, 6456 (1987).

${ }^{71}$ J. D. Honeycutt and H. C. Andersen, J. Phys. Chem. 91, 4950 (1987).

${ }^{72}$ P.-N. Roy, J. Chem. Phys. 119, 5437 (2003).

${ }^{73}$ D. J. Wales and R. S. Berry, J. Chem. Phys. 92, 4283 (1990).

${ }^{74}$ J. Jellinek, T. L. Beck, and R. S. Berry, J. Chem. Phys. 84, 2783 (1986)

${ }^{75}$ G. Franke, E. R. Hilf, and P. Borrmann, J. Chem. Phys. 98, 3496 (1993). 